\title{
Cellular Retention Assay to Determine the Interaction Frequency of CD19-directed Chimeric Antigen Receptor (CAR) Engineered Cells against CD19+ Leukemic Cells
}

\author{
Marvin I. De los Santos ${ }^{1,2, *}$ and Samuel D. Bernal ${ }^{2,3}$
}

\author{
${ }^{1}$ University of the Philippines, Diliman, Quezon City, Philippines; ${ }^{2}$ GlobeTek Science Foundation, Makati \\ City, Philippine; ${ }^{3}$ Cedars-Sinai Medical Center, Section of Hematology \& Oncology, Los Angeles, \\ California, USA \\ *For correspondence: midelossantos1215@gmail.com
}

\begin{abstract}
[Abstract] Cancer recognition by chimeric antigen receptor T (CAR-T) cell is prerequisite for cancer killing to occur. Anomalies in the binding specificity of scFv in the CAR construct may prevent successful eradication of these cancer cells. In some cases, these anomalies (i.e., altered specificity) may cause deleterious effects such as on-target off-tumor toxicity or on-set CAR-T cell activation that may lead to life-threatening complications. We describe in this assay an easy, flexible and cheap way of analyzing target specificity of CAR-engineered cells toward cancer in the context of cell-to-cell interaction that can be used to screen other antigen-specific CARs. We are coining this test as cellular retention assay.
\end{abstract}

Keywords: Cell-to-cell interaction assay, CAR-T cell, CD19 immunotherapy, ScFv affinity, Cellular retention

[Background] The encouraging clinical outcome endowed by CD19-directed chimeric antigen receptor $\mathrm{T}$ (CAR-T) cells in treating CD19+ hematologic malignancies motivated the development of more CART platforms for therapeutic application in wide variety of cancer types including solid tumors and other types of liquid cancers (Newick et al., 2016; Park et al., 2016). Chimeric antigen receptors (CARs) are composed of 1) an antigen-specific scFv (single chain variable fragment) moiety found at the extracellular matrix and 2) T cell activating intracellular domain. CARs are constructed by fusing a cancer antigen-specific scFv of an antibody molecule with $\mathrm{T}$ cell-associated activation domain such as CD3 zeta only or in combination with certain co-stimulatory molecules such as CD137 (4-1BB), CD28 and/or in the presence of inducible cytokines (Figure 4A). While the intracellular region facilitates $T$ cellmediated cancer killing, the ScFv domain of CAR performs the crucial role in the "search and attack" operation mounted by engineered T cells against cancer. Majority of pre-clinal workflow in CAR-T development relies heavily on characterizing the binding of $\mathrm{scFv}$ in unfused, CAR-independent context (such as in phage/ yeast display or produced from hybridoma technology) using several immuno-assays. Once the scFv is constructed and fused with the rest of the CAR expression cassette followed by T cell transduction or transfection, cytotoxicity and cancer killing in an in vitro co-culture challenge or xenogeneic mouse models are immediately performed without verifying the retained binding specificity of scFv in the CAR-fused form (Wang and Rivière, 2016; Levine et al., 2017; Vormittag et al., 2018).

Cancer cell recognition by CAR-T cell is a fundamental prerequisite for cancer killing to occur. Anomalies and defects in the binding specificity of ScFv in the CAR-fused form may prevent the efficient 
anti-cancer activity of these engineered cells and may instead cause detrimental side effects which include on-target off-tumor cytotoxicity and on-set CAR-T activation. Due to the complexity of CAR cloning and construction, along with the intricate and complicated antigen recognition mechanisms contributed by cancer pro-survival strategies, the poor and expensive structural elucidation within cellular environment and the unpredicted high toxicity rate of CAR-T therapy contributed by undesirable scFv characteristics (De los Santos and Bernal, 2018), the analysis of retained antigen specificity of scFv in the CAR-fused form is very crucial in the pre-clinal workflow of CAR-T development.

In this assay, we demonstrate the retainment of target specificity of CAR-engineered cell with cancer antigen in a cell-to-cell interaction assay (cellular retention assay). HEK293 cells are engineered to express the CD19-specific CAR (serving as CAR-engineered cells). An in-house established leukemic cell line established from cancer blood biopsy (Cayrefourcq et al., 2015), AMLK cells, was used as a cancer target model, bearing the CD19 antigen at the current passage. This cellular retention assay measures the ability of cell-to-cell mediated substrate-anchorage retention as a measure of CAR scFv binding to target cancer antigen as illustrated in Figure 1 below.

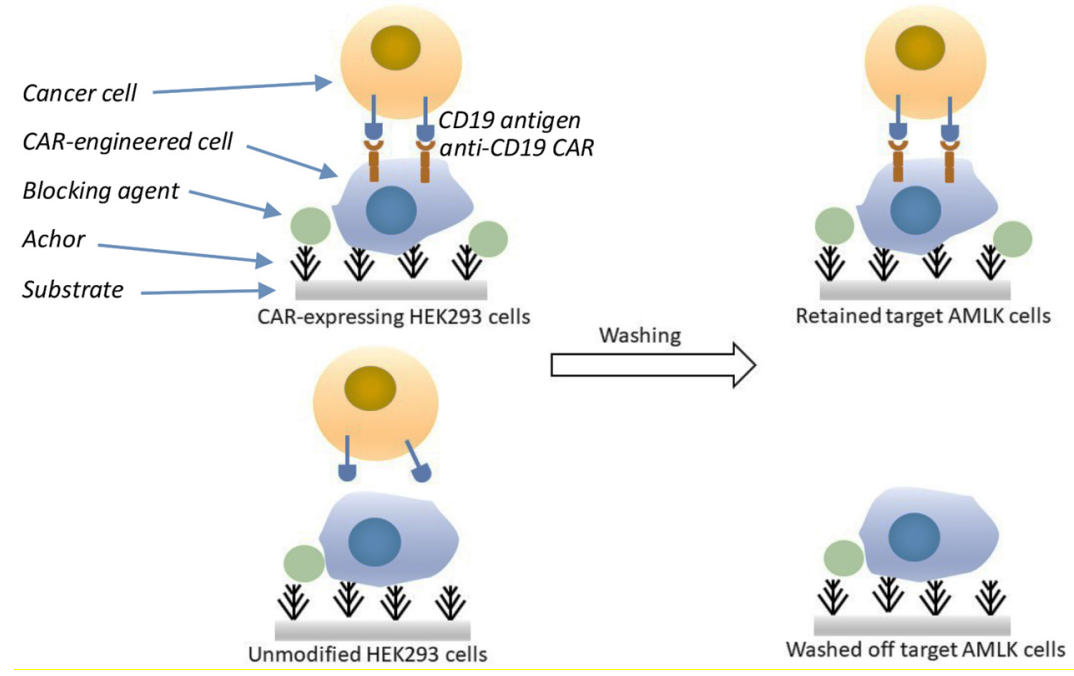

Figure 1. Cellular retention assay model to determine cell-to-cell mediated recognition of CD19 CAR engineered cells with CD19+ leukemic AMLK cells

\section{Materials and Reagents}

A. Cell culture expansion

1. $75 \mathrm{~cm}^{2}$ tissue culture flask (Corning, Falcon ${ }^{\circledR}$, catalog number: 353110 )

2. $25 \mathrm{~cm}^{2}$ tissue culture flask (Corning, Falcon ${ }^{\circledR}$, catalog number: 353108 )

3. $15 \mathrm{ml}$ conical tube (Fisher Scientific, Fisherbrand ${ }^{\mathrm{TM}}$, catalog number: 05-539-5)

4. Serological pipette $5 \mathrm{ml}$ (Corning, Stripette ${ }^{\mathrm{TM}}$, catalog number: 4487)

5. Serological pipette $10 \mathrm{ml}$ (Corning, Stripette ${ }^{\mathrm{TM}}$, catalog number: 4488)

6. HEK293 cells (ATCC, catalog number: CRL-1573) 
7. Target cancer cells (AMLK acute myeloid leukemia cells, Globetek Science Foundation Inc, Makati City, Philippines)

8. Dulbecco's Modified Eagle Medium (Mediatech, catalog number: 10-013-CV)

9. RPMI 1640 (Thermo Fisher Scientific, Gibco ${ }^{T M}$, catalog number: 11875093)

10. Heat inactivated fetal bovine serum (Sigma-Aldrich, catalog number: F4135)

11. Phosphate buffered saline, $1 \times$ (Mediatech, catalog number: 21-031-CV)

12. Sodium bicarbonate, 7.5\% (Thermo Fisher Scientific, Gibco, catalog number: 25080094)

13. Media 1 (see Recipes)

14. Media 2 (see Recipes)

B. Lipofection and CD19-CAR expression in HEK293 cells

1. 6-well plates, flat-bottom (Corning, Falcon ${ }^{\circledR}$, catalog number: 353046 )

2. $15 \mathrm{ml}$ conical tube (Fisher Scientific, Fisherbrand ${ }^{\mathrm{TM}}$, catalog number: 05-539-5)

3. $1.5 \mathrm{ml}$ Eppendorf microcentrifuge tubes (Sigma-Aldrich, catalog number: T9661-500EA)

4. Micropipette tips [Thermo Fisher Scientific, catalog numbers: TF102-20-Q (0.1-10 $\mu \mathrm{l})$; TF140-

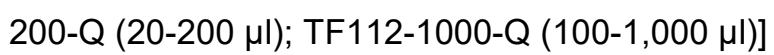

5. Phosphate buffered saline, $1 \times$ (Mediatech, catalog number: 21-031-CV)

6. CAR construct (anti-CD19 scFv-CD28-CD137-CD3z) and empty mammalian expression vector

7. Lipofectamine $2000^{\circledR}$ reagent (Thermo Fisher Scientific, Invitrogen ${ }^{\mathrm{TM}}$, catalog number: 11668027)

8. Opti-MEM ${ }^{\mathrm{TM}}$ I reduced serum medium (Thermo Fisher Scientific, Gibco ${ }^{\mathrm{TM}}$, catalog number: 11058021)

9. Membrane protein extraction kit such as ReadyPrep ${ }^{\mathrm{TM}}$ Extraction Kit- Membrane I (Bio-Rad, catalog number: 163-2088)

10. Qubit protein assay quantification kit (Thermo Fisher Scientific, Invitrogen ${ }^{\mathrm{TM}}$, catalog number: Q33211)

11. Laemmli sample buffer, $2 x$ (Bio-Rad, catalog number: 1610737)

12. 2-Mercaptoethanol (Sigma-Aldrich, catalog number: M6250-100ML)

13. NUPAGE ${ }^{T M}$ PAGE gels (Thermo Fisher Scientific, Invitrogen ${ }^{\mathrm{TM}}$, catalog number: NP0336BOX)

14. Precision Plus Protein ${ }^{\mathrm{TM}}$ All Blue Prestained Protein Standards (Bio-Rad, catalog number: 1610373)

15. Molecular biology grade water (Fisher Scientific, HyClone ${ }^{\mathrm{TM}}$, catalog number: $\mathrm{SH} 3053803$ )

16. MOPS (Fisher Scientific, Fisher BioReagents ${ }^{\top \mathrm{M}}$, catalog number: BP308-100)

17. Tris (Fisher Scientific, Fisher BioReagents ${ }^{\mathrm{TM}}$, catalog number: BP152-500)

18. EDTA (Fisher Scientific, Fisher Chemical ${ }^{\mathrm{TM}}$, catalog number: S316-212)

19. SDS (Fisher Scientific, Fisher BioReagents ${ }^{\mathrm{TM}}$, catalog number: BP166-100)

20. Coomasie Brilliant Blue R250 (Thermo Fisher Scientific, catalog number: 20278)

21. Glacial acetic acid (RCI Labscan, catalog number: AR1002-P2.5L)

22. Methanol (RCI Labscan, catalog number: AR1115-P4L) 
23. MOPS-SDS running buffer (see Recipes)

24. Coomasie protein stain (see Recipes)

25. Destaining solution (see Recipes)

C. Cellular retention assay

1. 24-well plates, flat-bottom (Corning, Falcon ${ }^{\circledR}$, catalog number: 353047 )

2. $1.5 \mathrm{ml}$ Eppendorf microcentrifuge tubes (Sigma-Aldrich, catalog number: T9661-500EA)

3. Micropipette tips [Thermo Fisher Scientific, catalog numbers: TF102-20-Q (0.1-10 $\mu \mathrm{l})$; TF140-

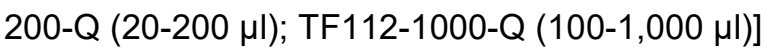

4. $15 \mathrm{ml}$ conical tube (Fisher Scientific, Fisherbrand ${ }^{\mathrm{TM}}$, catalog number: 05-539-5)

5. Countess cell counting chamber slides (Thermo Fisher Scientific, Invitrogen ${ }^{\mathrm{TM}}$, catalog number: C10228)

6. Poly-L-lysine (ScienceCell, catalog number: 0413)

7. Bovine serum albumin (Fisher Scientific, Fisher Bioreagents ${ }^{T M}$, catalog number: BP9703100)

8. Anti-CD19 antibody (Santa Cruz Biotechnology, IgG1 kappa light chain F-3 clone, catalog number: sc-373897)

9. Non-specific antibody, isotype control (Santa Cruz Biotechnology, IgG1 kappa light chain AT80 clone, catalog number: sc-58982)

10. Trypan blue stain, $0.4 \%$ (Thermo Fisher Scientific, Invitrogen ${ }^{\mathrm{TM}}$, catalog number: T10282)

11. Phosphate buffered saline, $1 \times$ (Mediatech, catalog number: 21-031-CV)

\section{Equipment}

1. Electric pipet controller (Fisher Scientific, Fisherbrand ${ }^{\mathrm{TM}}$, catalog number: 14-955-202)

2. Pipettes

3. Cell culture $\mathrm{CO}_{2}$ incubator (Nuaire, model number: $\mathrm{NU}-5100 \mathrm{E}$ )

4. Cell processing centrifuge (Hermle, Universal Centrifuge, model number: Z 366)

5. Microliter centrifuge (Thermo Fisher Scientific, SORVALL Legend Micro 21, model number: D37520)

6. BioSafety cabinet

7. Inverted microscope (ZEISS, Primo Vert, catalog number: 12-070-466)

8. PAGE system (Thermo Fisher Scientific, XCell SureLock ${ }^{\mathrm{TM}}$ Mini Cell, catalog number: EI0001)

9. Countess I automated cell counter (Thermo Fisher Scientific, Invitrogen ${ }^{\mathrm{TM}}$, catalog number: C10227)

\section{Software}

1. Office Excel (2003 or later versions, Microsoft) or any spreadsheet software 


\section{Procedure}

\section{A. Anti-CD19 CAR lipofection in HEK293}

Note: HEK293 cells are used as representative of CD19-specific CAR engineered cells and CD19+ AMLK leukemic cells are used as a cancer target for recognition by CAR-engineered cells. Researchers can use alternative cells based on their desired cancer antigen.

1. Culture HEK293 cells in $75 \mathrm{~cm}^{2}$ culture flasks using Media 1 and AMLK cells in $25 \mathrm{~cm}^{2}$ culture flask using Media 2; expand both cell lines until each yields $15 \times 10^{6}$ cells. Figure 2 shows the morphology of HEK293 and AMLK cells used in this experiment.

Note: An $80-90 \%$ confluency of HEK293 cells in a T75 flask is enough to yield the required cell count. AMLK cells grow in suspension and have doubling time of approximately 3 days. Based on the initial seeding density, calculate the number of days required to obtain the desired cell quantity. Continue expanding AMLK cells until further use.
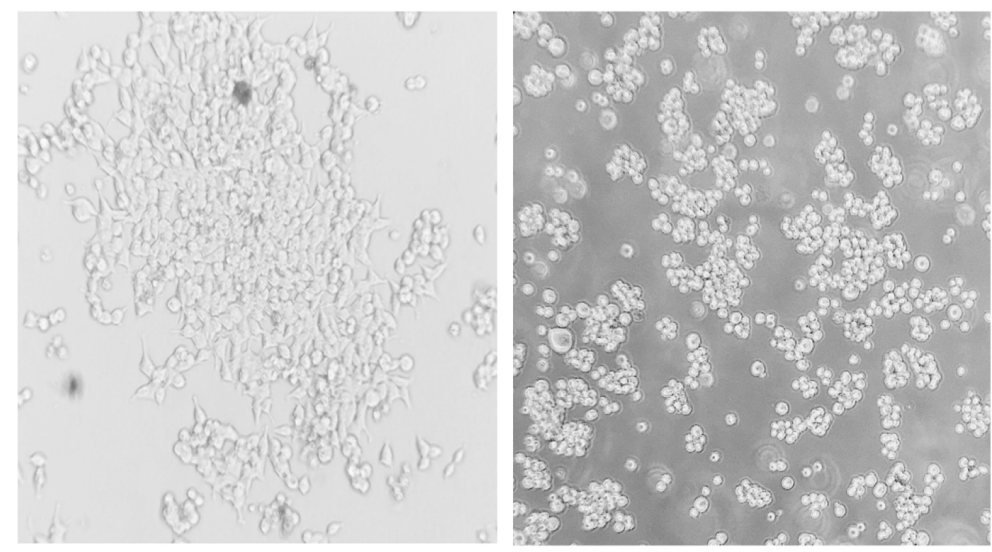

Figure 2. Photomicrographs of HEK293 cells (left) and AMLK cells (right). Magnified 100x.

2. Harvest HEK293 cells in $15 \mathrm{ml}$ conical tube and collect the cell pellet by centrifugation at $500 x$ $g$ for $5 \mathrm{~min}$ at room temperature.

Note: Some HEK293 cells can be dislodged from culture substrate using PBS only (without trypsin or EDTA) by incubation for $1 \mathrm{~min}$ at $37^{\circ} \mathrm{C}$ followed by gentle tapping on the side of the flask.

3. Discard the supernatant and resuspend cells in $1 \times 10^{6} \mathrm{cell} / \mathrm{ml}$ of Media 1. Plate approximately $1 \times 10^{6}$ cells or $1 \mathrm{ml}$ of resuspended cells in each well of three 6-well plates (see Table 1). Fill each well with $1 \mathrm{ml}$ more of Media 1 to feed the cells at a final volume of $2 \mathrm{ml} /$ well. Incubate overnight at $37^{\circ} \mathrm{C}$ with $5 \% \mathrm{CO}_{2}$. 
Table 1. Treatment and controls for HEK293 cell lipofection

\begin{tabular}{lll}
\hline Vector & Purpose & Plate \\
\hline anti-CD19 scFv-CD28-CD137-CD3z CAR & CD19-specific CAR & Plate 1 \\
Empty plasmid vector & Transfection control & Plate 2 \\
Without vector & Negative control & Plate 3 \\
\hline
\end{tabular}

4. Optionally, media from the culture plates after overnight incubation can be replaced with $2 \mathrm{ml}$ of fresh Media 1. Keep the cells in the incubator until further use.

5. Prepare plasmid vector and Lipofectamine $2000^{\circledR}$ mix on ice based on Table 2 below.

Table 2. Plasmid vector and Lipofectamine $2000^{\circledR}$ preparation

\begin{tabular}{lllll}
\hline \multirow{2}{*}{ Components } & \multicolumn{2}{l}{${ }^{1}$ Culture Plates } & \multirow{2}{*}{ Tube } \\
\cline { 2 - 4 } & Plate 1 & Plate 2 & Plate 3 & \\
\hline${ }^{2}$ Vector DNA & $15 \mu \mathrm{g}$ & $15 \mu \mathrm{g}$ & - & \multirow{2}{*}{ Tube 1 } \\
Opti-MEM $^{\mathrm{TM}}$ & $750 \mu \mathrm{l}$ & $750 \mu \mathrm{l}$ & $750 \mu \mathrm{l}$ & \\
${ }^{3}{ }^{2}$ ipofectamine $2000^{\circledR}$ & $50 \mu \mathrm{l}$ & $50 \mu \mathrm{l}$ & $50 \mu \mathrm{l}$ & Tube 2 \\
Opti-MEM ${ }^{\mathrm{TM}}$ & $700 \mu \mathrm{l}$ & $700 \mu \mathrm{l}$ & $700 \mu \mathrm{l}$ & \\
\hline
\end{tabular}

${ }^{1}$ Three sets should be prepared separately based on vector treatment indicated in Table 1.

${ }^{2}$ The plasmid (vector) DNA can be prepared as lyophilized or resuspended in TE buffer not more than $50 \mu \mathrm{l}$.

${ }^{3}$ Mix the Lipoefectamine reagent well before use.

6. Vortex Tubes 1 and 2 for $10 \mathrm{~s}$ and spin down for $5 \mathrm{~s}$ to recollect the solution. Incubate the tubes at room temperature for $5 \mathrm{~min}$. Proceed to the next step within $25 \mathrm{~min}$ for optimal lipofection.

7. Combine tubes 1 (opti-MEM/DNA) and 2 (Opti-MEM/lipofectamine) to a final volume of $1.5 \mathrm{ml}$ and mix gently by vortexing. Incubate further at room temperature for $20 \mathrm{~min}$.

8. Pipette $250 \mu \mathrm{l}$ of DNA/lipofectamine complex into each well of the 6-well plate per vector treatment indicated in Table 1. Mix the plate by gently by rocking the plates back and forth 10 times.

9. Incubate cells at $37{ }^{\circ} \mathrm{C} 5 \% \mathrm{CO}_{2}$ for $48 \mathrm{~h}$ prior to testing for CAR expression.

10. Optionally, in the absence of fluorescence reporter to detect transfection efficiency (especially when developing non-GFP vectors for future therapeutic use), transgene copy number can be determined by qPCR as shown in Figure 3 below.

Note: It is always a best practice to determine transfection efficiency before proceeding with any post-transgenesis assays. For qPCR-based plasmid copy number identification, usually the transfected cells are lysed, and total genomic DNA is isolated. Primer pair specific to the vector and CAR sequence is usually used to detect the presence of transgenesis. Quantification can be measured by using a reference plasmid standard with known concentration or copy number count prepared in three 10-fold dilutions. Copy number quantification can then be derived by 
using the $y$ value of the standard curve or can be automatically generated using qPCR systems (Cohen et al., 2009; Sommeregger et al., 2013).
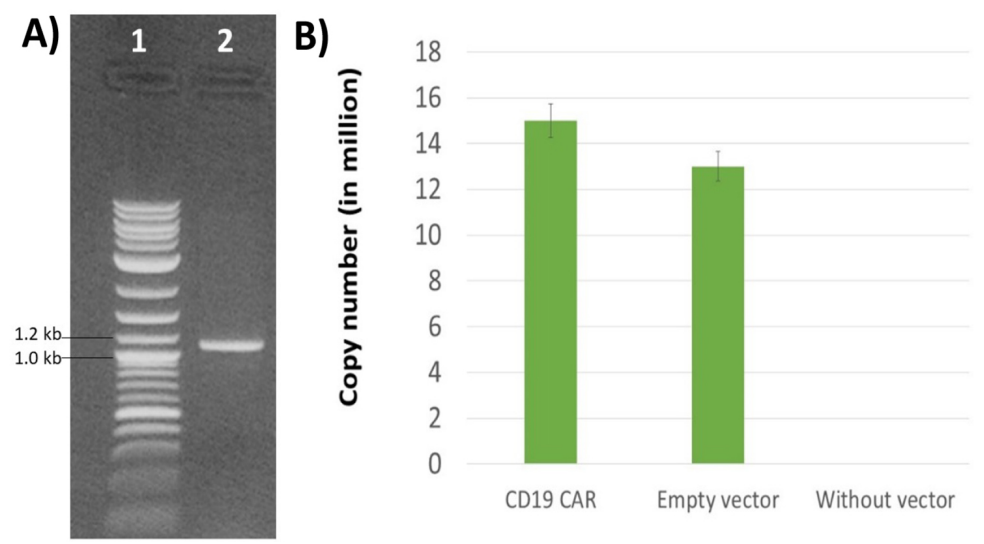

Figure 3. Determination of sample transgene copy number in HEK293 cells. A. Showing optimized PCR amplification of the intracellular signaling domain of CAR construct. Lane 1: molecular weight marker; lane 2: CAR-transfected HEK293 cells. B. Plasmid copy number quantification by qPCR (Sommeregger et al., 2013) using vector-specific primer, copy number in million per 1 million of transfected HEK293 cells. Copy number ratio in this experiment is 15 million per 1 million of CD19 CAR engineered HEK 293 cells (15:1 ratio) and 13 million per 1 million empty vector-transfected HEK293 cells (13:1 ratio) with no detectable transgenesis in without vector cells (negative control), confirming successful transfection with high efficiency.

B. Confirmation of successful transfection and CAR membrane expression

1. Harvest cells in one well of each culture plate, preferably by scraping to avoid disturbing cells from other wells.

2. Pellet down the cells by centrifugation at $500 \times g$ for $5 \mathrm{~min}$. Remove supernatant and wash with PBS once.

3. Resuspend pellet and isolate membrane proteins using ReadyPrep ${ }^{\mathrm{TM}}$ Protein Extraction Kit (Membrane I) or any alternative buffers and method.

Note: Chimeric antigen receptors (CARs) are supposed to be sorted out as transmembrane protein. Preferentially isolating membrane-bound proteins will make the analysis of transgene expression easier and validate proper sorting of proteins in the cell line model shown in Figure 4. 
A

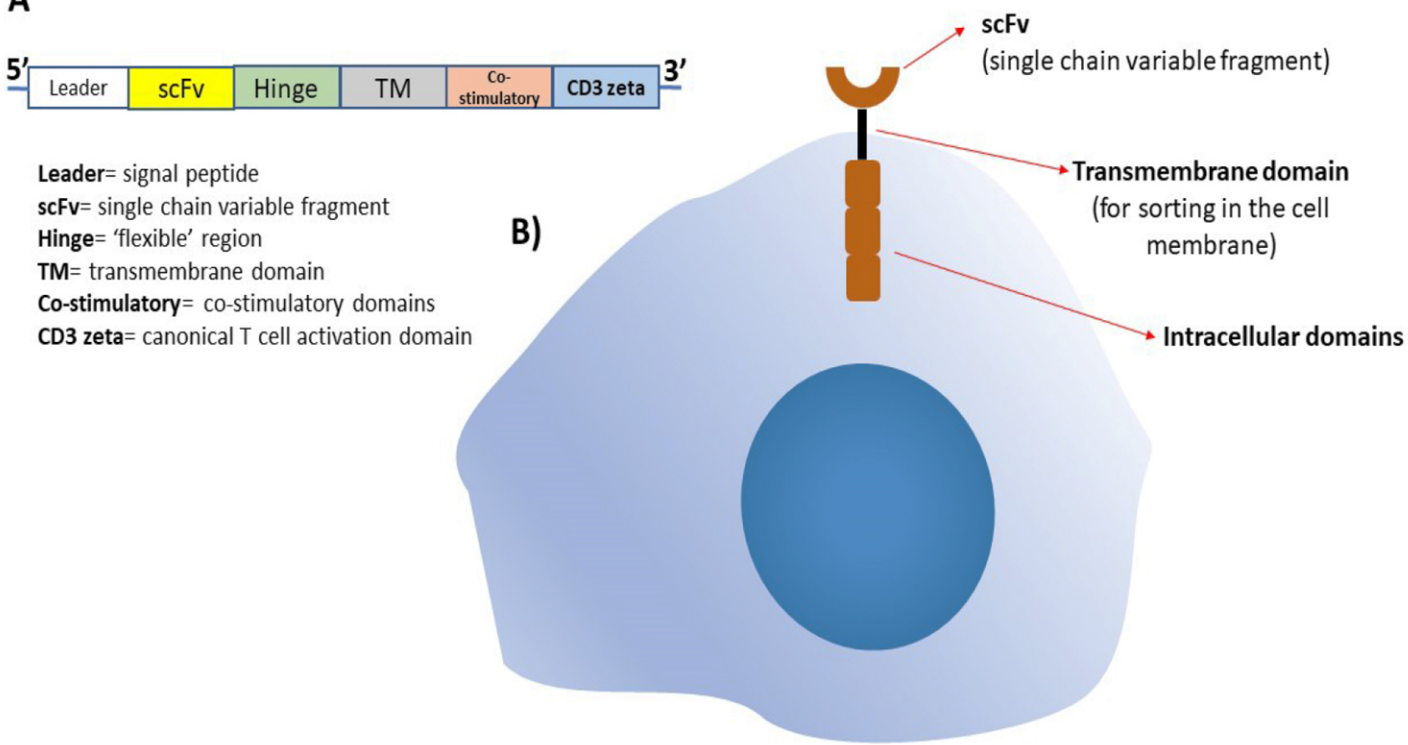

Figure 4. CAR sorting in cell membrane. A. Schematic of a typical CAR construct. B. Expression and sorting of CAR protein in the cell membrane of engineered HEK293.

4. Make three two-fold dilutions of the membrane protein extract starting from $30 \mu \mathrm{g}, 15 \mu \mathrm{g}$ and $7.5 \mu \mathrm{g}$ with PBS and mix with 1:1 ratio of 2x Laemmli buffer containing 5\% 2-Mercaptoethanol. Boil the samples for $20 \mathrm{~min}$.

5. Load the samples and protein ladder in polyacrylamide gel noting the orientation of each sample.

6. Run the proteins in appropriate conditions (MOPS-SDS buffer for NuPAGETM gels at $250 \mathrm{~V}$ for $30 \mathrm{~min}$ )

7. After the run, wash the gel by soaking in water for $3 \mathrm{~min}$.

8. Stain the protein by submerging the gel in Coomasie solution and boil for $1 \mathrm{~min}$. Incubate at room temperature for $30 \mathrm{~min}$ in the dark followed by washing with water for $2 \mathrm{~min}$.

Note: The Coomasie blue can be preheated to boiling in a $100{ }^{\circ} \mathrm{C}$ hot plate prior to staining. Boiling can be simultaneously done with the gel soaked in using a microwave oven.

9. Visualize protein bands by soaking the gel in excess destaining solution overnight.

10. Analyze protein expression as shown in Figure 5.

Note: If an antibody is available for your CAR protein, proceed with western blot. 


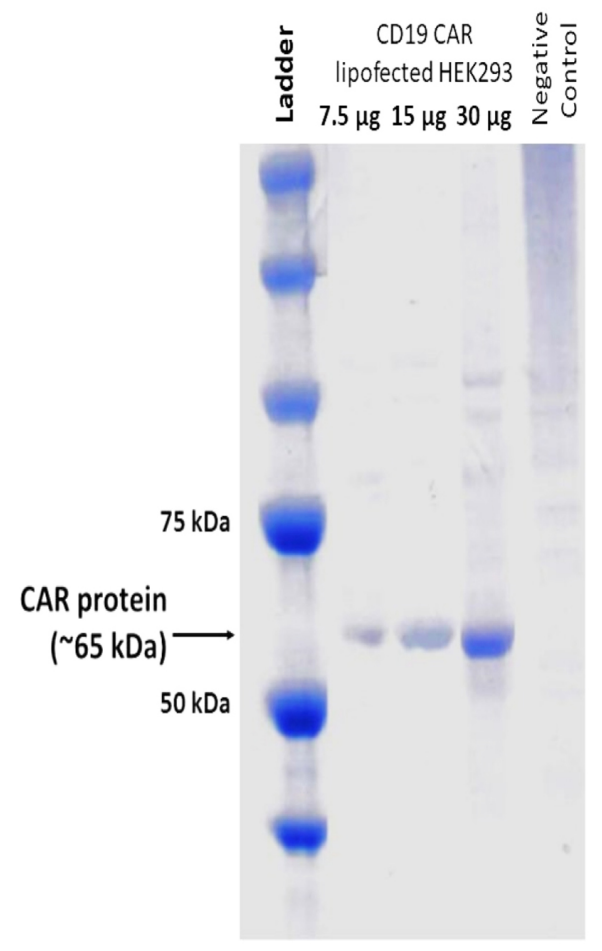

Figure 5. Representative polyacrylamide gel electrophoresis profile of CAR lipofected HEK293 cells. Presence of $65 \mathrm{kDa}$ band size indicates successful transgene expression in HEK 293 cells lipofected with CAR construct; the band thickness increases with increasing sample concentration. Whereas, negative control set-up (HEK293 cells lipofected with empty vector) did not show this $65 \mathrm{kDa}$ size band.

C. Retention assay

1. Coat one 24-well plate with $50 \mu \mathrm{g} / \mathrm{ml}$ of poly-L-lysine (usually $500 \mu \mathrm{l}$ can cover the whole well surface) at room temperature for $1 \mathrm{~h}$.

2. Rinse and wash the wells with PBS twice.

3. Harvest $1 \times 10^{6}$ lipofected HEK293 cells and resuspend in PBS.

4. Seed $1 \times 10^{5}$ cells in each well following assay format in Figure 6 below. Allow the cells to attach on the surface for $5 \mathrm{~min}$ on ice.

Note: The format shown in Figure 6 represents a duplicate assay, replication can be increased to the desired number. 


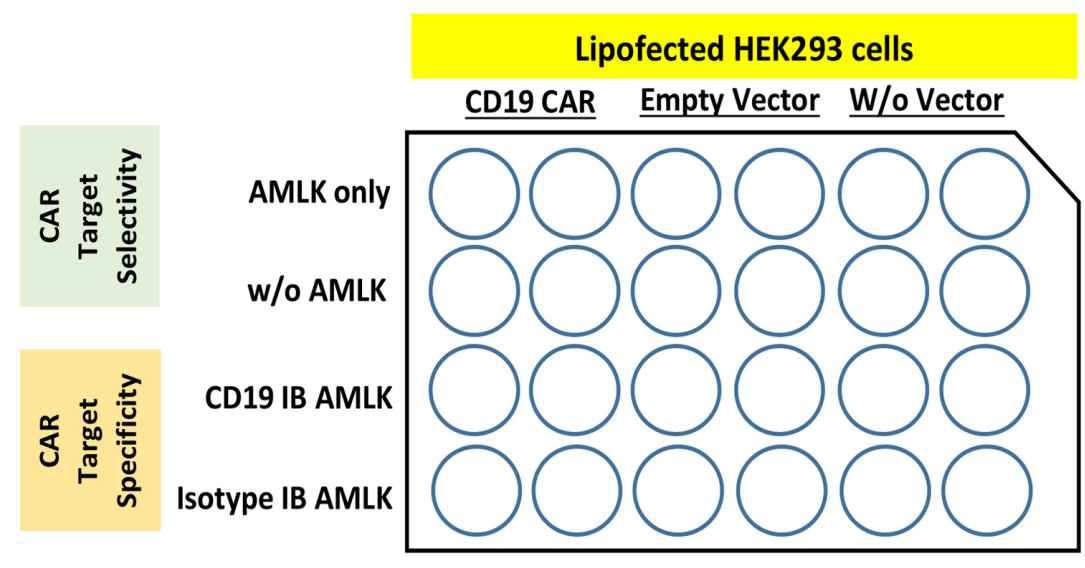

Figure 6. Retention assay format in 24-well plate (represented in duplicate). IB= immunoblocked with antibody.

5. Block any remaining attachment site on poly-L-lysine by adding BSA (bovine serum albumin) at a final concentration of $2 \%$ using PBS in each well. Incubate on ice for $5 \mathrm{~min}$.

6. While blocking, harvest $5 \times 10^{6} \mathrm{AMLK}$ cells from culture by centrifugation at $800 \times \mathrm{g}$ for $3 \mathrm{~min}$.

7. Divide the cells into 3 categories for treatments: AMLK only, CD19 IB AMLK and Isotype IB AMLK.

a. For AMLK only, seed 1:1 lipofected HEK:target AMLK ratio that is $1 \times 10^{5}$ AMLK cells per well.

b. For C19 IB AMLK, immuno-block target antigen by pre-incubating $200 \mathrm{ng}$ of anti-CD19 antibody per $1 \times 10^{6}$ AMLK cells for 10 min on ice in a maximum volume of $0.5 \mathrm{ml}$ PBS. Seed 1:1 lipofected HEK:target AMLK ratio per well ( $1 \times 10^{5}$ CD19 IB AMLK cells).

c. For isotype IB AMLK, same parameters as in Step C7b using isotype control antibody. Seed 1:1 lipofected HEK:target AMLK ratio per well ( $1 \times 10^{5}$ isotype IB AMLK cells).

d. For without AMLK treatments, use PBS only with the same volume as in Step C7a.

8. Allow cell-to-cell interaction by incubating the cells for $10 \mathrm{~min}$ on ice.

Note: The total volume during this retention assay should not exceed $0.5 \mathrm{ml}$.

9. Pipette out the supernatant carefully from the side of the well.

10. Wash the cells carefully with $1 \mathrm{ml}$ of PBS, twice to achieve retention profile as shown in Figure 7 below.

Note: Wash the cells only for $10 \mathrm{~s}$ and dispense the liquid at the side of the well to prevent introducing physical disruption to the binding of the cells. 

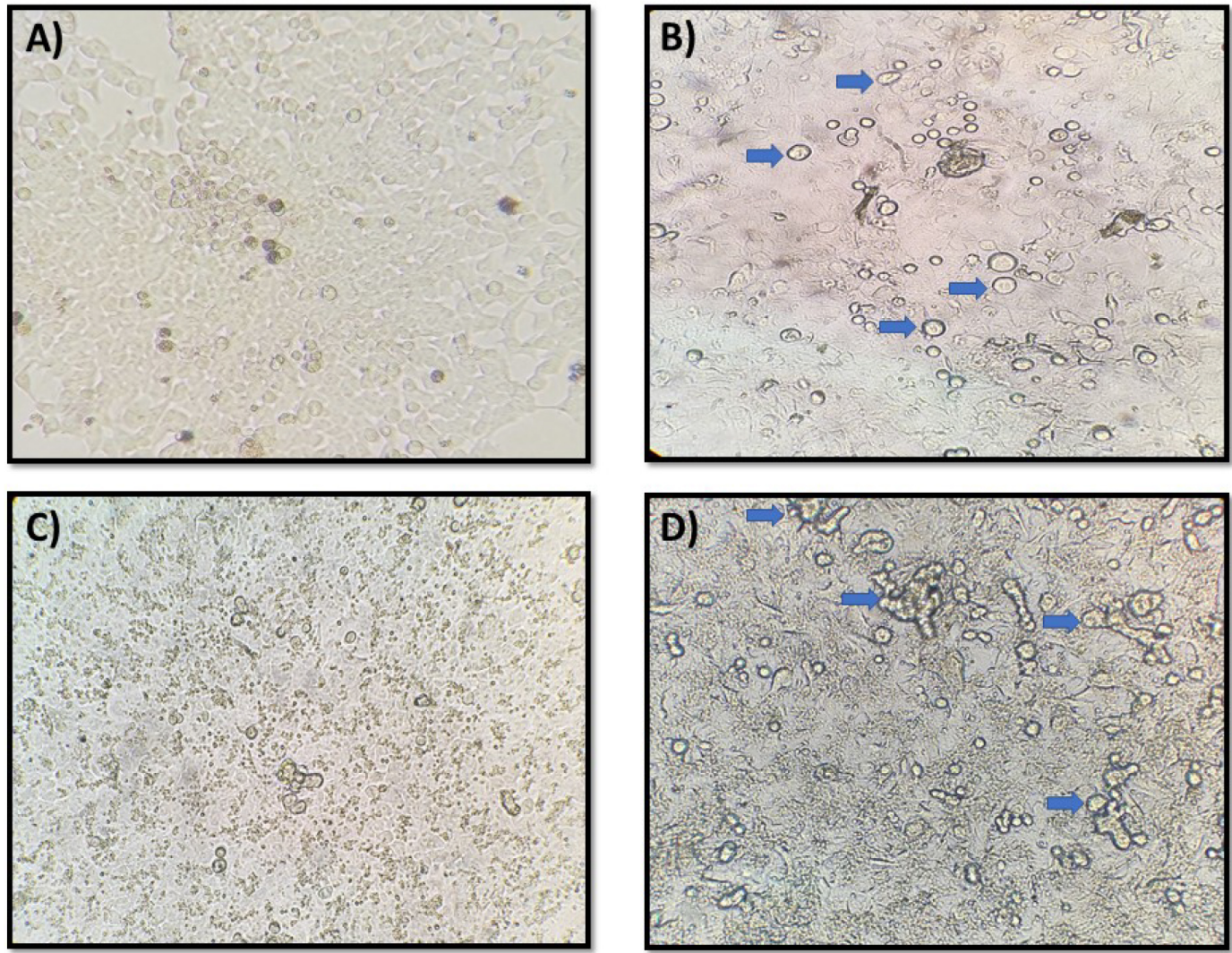

Figure 7. Retention profile of AMLK cells on substrate-anchored CAR-engineered HEK293 cells. A. Without AMLK treatment. B. AMLK only. C. CD19 IB AMLK. D. Isotype IB AMLK. Blue arrows represent AMLK cells on the surface of HEK293 cells, indicative of retention due to binding of CD19 scFv on HEK293 with CD19 antigen on AMLK cells. Samples were magnified 100x.

11. Resuspend the cells in $100 \mu \mathrm{l}$ of PBS. Detach the cells by agitating the plate back and forth.

12. Transfer $10 \mu \mathrm{l}$ into a new tube and add $10 \mu \mathrm{l}$ of $0.4 \%$ Trypan blue stain. Mix well to dissociate cells and transfer $10 \mu$ into Countess ${ }^{\mathrm{TM}}$ cell counting chamber slides.

13. Gate retained AMLK cells based on size parameters. HEK293 cells are usually measured at 11 to 15 microns while AMLK cells appear at 5 to 10 microns. Set the gating conditions to 5 microns as the minimum and 10 microns as the maximum to preferentially count retained AMLK target cells. Higher count indicates retention of cancer cells due to binding of CD19 antigen to the scFv of anchored HEK293 cells.

Note: Gate cell size based on specific cell diameter or size of your target cells.

14. Monitor total cell concentration.

D. Reverse retention assay

1. Coat 24 -well plate with $50 \mu \mathrm{g} / \mathrm{ml}$ of poly-L-lysine (usually $500 \mu \mathrm{l}$ can cover the whole well surface) at room temperature for $1 \mathrm{~h}$.

2. Rinse and wash the wells with PBS twice. 
3. Harvest $5 \times 10^{6}$ AMLK cells and resuspend in PBS.

4. Divide the cells into 3 categories for treatments: AMLK only, CD19 IB AMLK and Isotype IB AMLK.

a. For AMLK only, seed $1 \times 10^{5}$ AMLK cells per well.

b. For C19 IB AMLK, immuno-block target antigen by pre-incubating $200 \mathrm{ng}$ of anti-CD19 antibody per $1 \times 10^{6}$ AMLK cells for $10 \mathrm{~min}$ on ice in a maximum volume of $0.5 \mathrm{ml}$ PBS. Seed $1 \times 10^{5}$ CD19 IB AMLK cells per well.

c. For isotype IB AMLK, same parameters as in Step C7b using isotype control antibody. Seed $1 \times 10^{5}$ isotype IB AMLK cells per well.

d. For without AMLK treatments, use PBS only with the same volume as in Step D4a.

5. Block any remaining attachment site on poly-L-lysine by adding BSA (bovine serum albumin) at a final concentration of $2 \%$ in each well. Incubate on ice for $5 \mathrm{~min}$.

6. Harvest and seed $1 \times 10^{5}$ lipofected HEK293 cells in each well following assay format in Figure 6 above. Allow the cells to attach on the surface for $5 \mathrm{~min}$ on ice.

7. Allow cell-to-cell interaction by incubating the cells for $10 \mathrm{~min}$ on ice.

Note: The total volume during this retention assay should not exceed $0.5 \mathrm{ml}$.

8. Pipette out the supernatant carefully from the side of the well.

9. Wash the cells carefully with $1 \mathrm{ml}$ of PBS, twice.

Note: Wash the cells only for $10 \mathrm{~s}$ and dispense the liquid at the side of the well to prevent introducing physical disruption to the binding of the cells.

10. Resuspend the cells in $100 \mu \mathrm{l}$ of PBS. Detach the cells by agitating the plate back and forth.

11. Transfer $10 \mu \mathrm{l}$ into a new tube and add $10 \mu \mathrm{l}$ of $0.4 \%$ Trypan blue stain. Mix well and transfer $10 \mu$ into Countess ${ }^{\mathrm{TM}}$ cell counting chamber slides.

12. Gate retained HEK293 cells based on size parameters. HEK293 cells are usually measured at 11 to 15 microns while AMLK cells appear at 5 to 10 microns. Set the gating conditions to 11 microns as the minimum and 15 microns as the maximum to preferentially count retained HEK293 cells. Higher count indicates retention of HEK293 cells due to binding of scFv to CD19 of anchored AMLK cells.

Note: Gate cell size based on specific cell diameter or size of your target cells.

13. Monitor total cell concentration.

\section{Data analysis}

In this assay, the isotype IB set-up is used in order to measure 1) non-specificity binding of the CD19 IB set-up (as there has been an addition of antibody in the culture, serving as cross reactivity reference); 2) Fc-mediated binding of AMLK cells (to rule out that the retention in CD19 IB set-up was not due to the $\mathrm{Fc}$ recognition of the CD19 positive cells by the CAR-engineered cells, ruling out false positive count). 
AMLK only set-up measures the cell-to-cell interaction or retention profile of CD19 CARengineered HEK293 cells with CD19+ AMLK cells (Figure 9), and then "If the retention in CD19 IB is low then we are sure that the scFv in CAR has retained specificity to CD19.
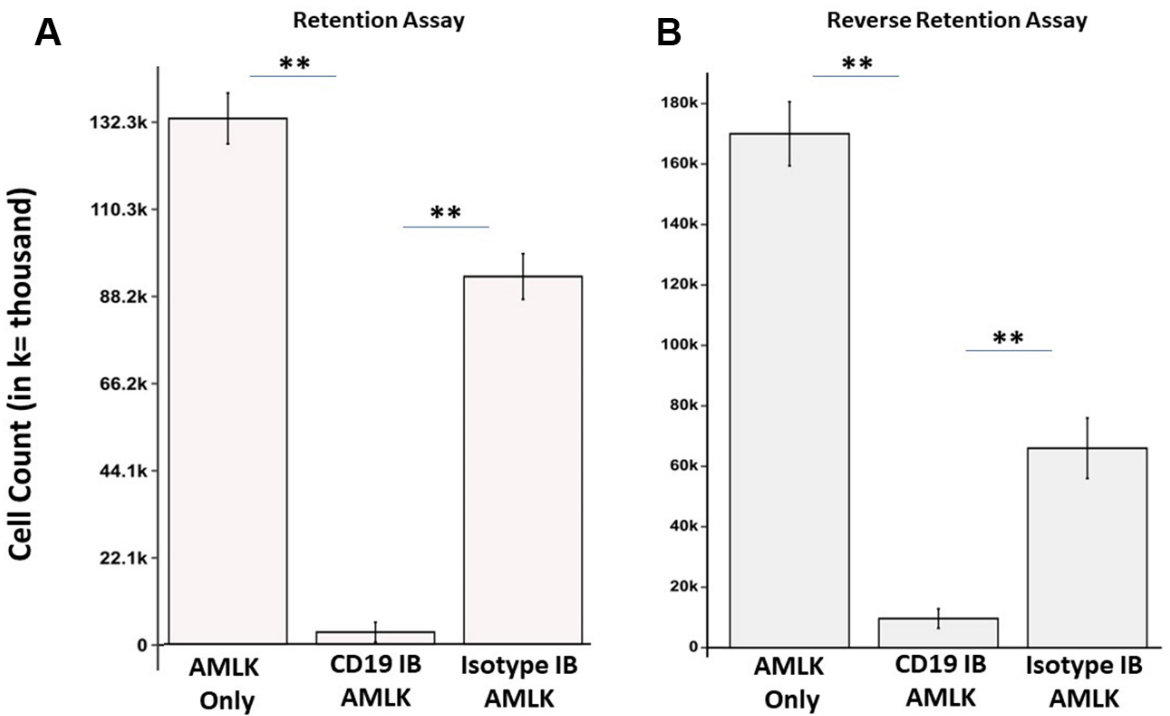

Figure 8. Statistical analyses of normalized gated cell count of cellular retention assay (A) and reverse retention assay (B). P-value less than 0.01 is considered statistically significant $\left({ }^{* *}\right)$.
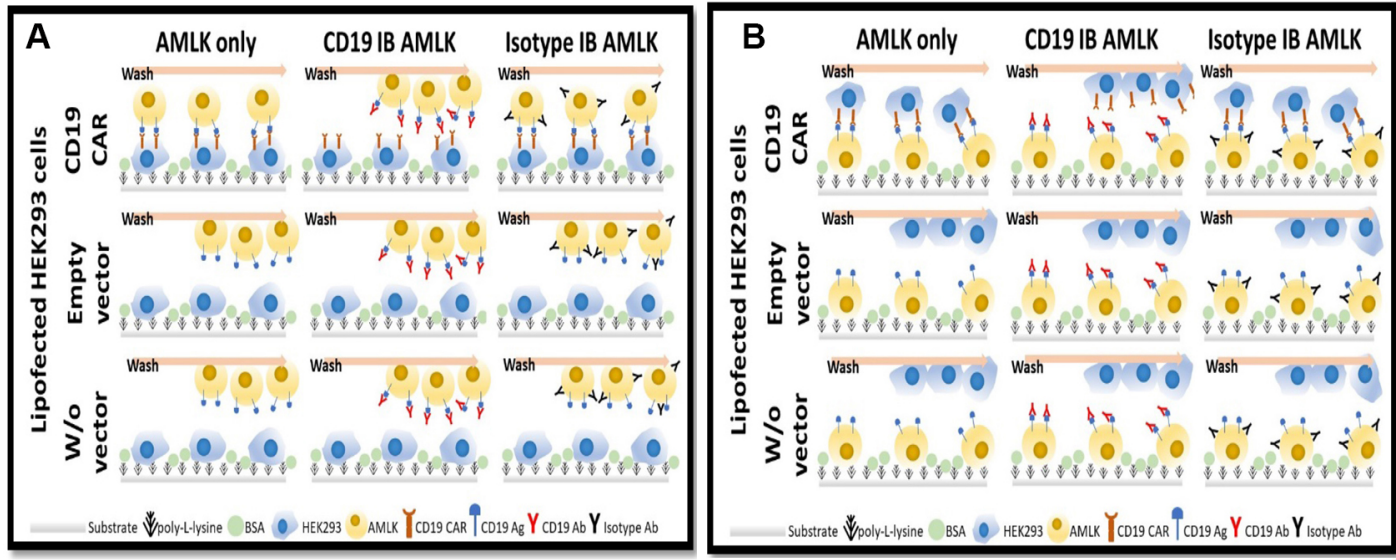

Figure 9. Schematic of treatments and mechanism of retention assay (A) and reverse retention assay $(B)$ 
1. Plot the total cell count using spreadsheet software (sample data in Table 3). Or you may copy the cell count report generated by the counting machine.

2. Normalize total cell count of all treatments over the count in 'without AMLK treatment' as seen in Table 3 .

3. Analyze the retention profile statistically by ANOVA (analysis of variance) with post-hoc Turkey HSD test to compare differences in gated normalized total cell counts (Figure 8). Retained CAR selectivity and specificity towards target antigen is indicated by higher retention count in AMLK only and Isotype IB AMLK treatments compared to CD19 IB AMLK $\left(P\right.$-value $\left.<0.01,{ }^{* *}\right)$.

Note: AMLK only or CD19 IB can be considered as relative retention count as long as each yields a statistically desired result for your analysis.

Table 3. Retention profile sample raw and normalized data

\begin{tabular}{|c|c|c|c|c|c|c|c|c|c|c|}
\hline & & \multicolumn{5}{|c|}{ Raw Data } & \multicolumn{4}{|c|}{ Normalized Data } \\
\hline \multirow{6}{*}{ 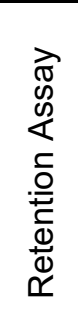 } & & & AMLK & CD19 IB & Isotype IB & Without & & AMLK & CD19 IB & Isotype IB \\
\hline & & Replication & Only & AMLK & AMLK & AMLK & Replication & Only & AMLK & AMLK \\
\hline & & R1 & 208000 & 76000 & 168000 & 75000 & R1 & 133000 & 1000 & 93000 \\
\hline & & $\mathrm{R} 2$ & 220000 & 95000 & 182000 & 89000 & $\mathrm{R} 2$ & 131000 & 6000 & 93000 \\
\hline & & R3 & 226000 & 86000 & 166000 & 83000 & R3 & 143000 & 3000 & 83000 \\
\hline & & Mean & 218000 & 85667 & 172000 & 82333 & Mean & 135667 & 3333 & 89667 \\
\hline \multirow{6}{*}{\multicolumn{2}{|c|}{ 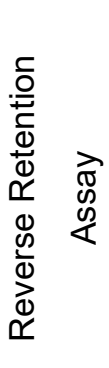 }} & & AMLK & CD19 IB & Isotype IB & Without & & AMLK & CD19 IB & Isotype IB \\
\hline & & Replication & Only & AMLK & AMLK & AMLK & Replication & Only & AMLK & AMLK \\
\hline & & $\mathrm{R} 1$ & 235000 & 75000 & 125000 & 69000 & $\mathrm{R} 1$ & 166000 & 6000 & 56000 \\
\hline & & $\mathrm{R} 2$ & 250000 & 99000 & 154000 & 88000 & $\mathrm{R} 2$ & 162000 & 11000 & 66000 \\
\hline & & R3 & 265000 & 95000 & 159000 & 83000 & R3 & 182000 & 12000 & 76000 \\
\hline & & Mean & 250000 & 89667 & 146000 & 80000 & Mean & 170000 & 9667 & 66000 \\
\hline
\end{tabular}


In this proposed protocol, the use of anti-CD19 antibody that competitively binds CAR target antigen and the use of isotype antibody will give an idea whether the specificity of scFv is lost or retained. If the specificity is retained (low retention count in CD19 IB) then the construct can be utilized for succeeding assays. But if the specificity is lost (high retention count in CD19 IB) then additional criteria on scFv selection may be administered (as reviewed in Delos Santos \& Bernal, 2018).

\section{$\underline{\text { Notes }}$}

1. Treatments without AMLK cells serve as a reference control for normalization. CD19 immunoblocked (IB) AMLK cells serve as an ScFV specificity control, wherein, the initial binding of antibody to the CD19 antigen on AMLK precludes the recognition of this antigen by CD19 CAR on HEK 293 cells leading to reduced retention/cell count (Figure 8). Isotype immunoblocked (IB) AMLK serves as an isotype control for non-specific binding to other antigens found in AMLK cells or antibody cross-reactivity with HEK293 cells. Low retention count of CD19 IB and high retention of AMLK only indicate the presence of CD19 specificity by CAR.

2. The advantage of using poly-L-lysine coated substrate is to prevent the cells from spontaneously dislodging from the substrate. Skipping this step will make the experiment fail. During long incubations, it is important to work on ice to slow down or delay cell death as it may affect the binding of the cells and their retention count. Since the retention data is derived from washing off non-binding cells, it is important to always slowly dispense and withdraw washing solution on the side of the well plate to avoid disturbing cell-to-cell binding. Also, make sure to prevent tapping or shaking the well plate to avoid loss of cell count. When using this protocol to validate other antigen-specific CARs, it is very important as well to validate whether the antibody binds native protein fold.

\section{$\underline{\text { Recipes }}$}

1. Media 1

$500 \mathrm{ml}$ DMEM Medium (with sodium bicarbonate and sodium pyruvate)

$50 \mathrm{ml}$ heat-inactivated fetal bovine serum

Keep at $4{ }^{\circ} \mathrm{C}$

2. Media 2

500 ml RPMI 1640 Medium (with L-glutamine)

$50 \mathrm{ml}$ heat-inactivated fetal bovine serum

$5 \mathrm{ml}$ sodium bicarbonate

Keep at $4{ }^{\circ} \mathrm{C}$

3. MOPS-SDS Running buffer $(1 \mathrm{~L})$ 
$10.46 \mathrm{~g}$ MOPS (50 mM)

$6.057 \mathrm{~g}$ Tris $(50 \mathrm{mM})$

$0.3 \mathrm{~g}$ EDTA $(1.025 \mathrm{mM})$

$1.0 \mathrm{~g}$ SDS $(1 \%)$

4. Coomasie protein stain $(100 \mathrm{ml})$

$0.25 \mathrm{~g}$ Coomasie Brilliant Blue R250 (0.25\%)

$10 \mathrm{ml}$ glacial acetic acid (10\%)

$90 \mathrm{ml}$ of $1: 1$ methanol to water ratio

5. Destaining solution $(100 \mathrm{ml})$

$10 \mathrm{ml}$ glacial acetic acid (10\%)

$50 \mathrm{ml}$ methanol $(50 \%)$

$40 \mathrm{ml}$ water $(40 \%)$

\section{Acknowledgments}

This work was internally supported by GlobeTek Science Foundation and Globetek Training Institute. We thank some of our lab members, Kristin Glori Pusing and Nemuel Kho for technical assistance.

\section{Competing interests}

The authors declare no conflict of interest.

\section{References}

1. De los Santos, M. I. and Bernal, S. D. (2018). CAR-T therapy for solid tumors: development of new strategies. Trends Immunotherapy 2: doi:10.24294/ti.v2i3.1064.

2. Levine, B. L., Miskin, J., Wonnacott, K. and Keir, C. (2017). Global manufacturing of CAR T cell therapy. Mol Ther Methods Clin Dev 4: 92-101.

3. Newick, K., Moon, E. and Albelda, S. M. (2016). Chimeric antigen receptor T-cell therapy for solid tumors. Mol Ther Oncolytics 3: 16006. doi:10.1038/mto.2016.6.

4. Park, J. H., Geyer, M. B. and Brentjens, R. J. (2016). CD19-targeted CAR T-cell therapeutics for hematologic malignancies: interpreting clinical outcomes to date. Blood 127(26): 3312-3320.

5. Sommeregger, W., Prewein, B., Reinhart, D., Mader, A. and Kunert, R. (2013). Transgene copy number comparison in recombinant mammalian cell lines: critical reflection of quantitative realtime PCR evaluation. Cytotechnology 65(5): 811-818.

6. Vormittag, P., Gunn, R., Ghorashian, S. and Veraitch, F. S. (2018). A guide to manufacturing CAR T cell therapies. Curr Opin Biotechnol 53: 164-181.

7. Wang, X. and Rivière, I. (2016). Clinical manufacturing of CAR T cells: foundation of a promising therapy. Mol Ther Oncolytics 3: 16015. 
8. Cayrefourcq, L., Mazard, T., Joosse, S., Solassol, J., Ramos, J., Assenat, E., Schumacher, U., Costes, V., Maudelonde, T., Pantel, K. and Alix-Panabieres, C. (2015). Establishment and characterization of a cell line from human circulating colon cancer cells. Cancer Res 75(5): 892901.

9. Cohen, R. N., van der Aa, M. A., Macaraeg, N., Lee, A. P. and Szoka, F. C., Jr. (2009). Quantification of plasmid DNA copies in the nucleus after lipoplex and polyplex transfection. $J$ Control Release 135(2): 166-174.

10. Sommeregger, W., Prewein, B., Reinhart, D., Mader, A. and Kunert, R. (2013). Transgene copy number comparison in recombinant mammalian cell lines: critical reflection of quantitative realtime PCR evaluation. Cytotechnology 65(5): 811-818. 